\title{
Letter to the Editor: Identifying and Addressing Barriers to Transgender Healthcare: Where We Are and What We Need to Do About It
}

\author{
Christopher K. Wong, BA, BS ${ }^{\top}$, Shefali P. Jain, BS², and Matthew Van de Graaf, BS ${ }^{3}$
}

J Gen Intern Med 37(4):972

DOI: $10.1007 / \mathrm{s} 11606-021-07267-6$

(c) Society of General Internal Medicine 2021

W e applaud the article by Warner and Mehta to bring attention to structural, interpersonal, and anticipation barriers to healthcare for transgender and non-binary (TNB) individuals. ${ }^{1}$ As medical student advocates for LGBTQ+ health, we agree that undergraduate medical education falls short in preparing future physicians to address the specific health needs of this community; a 2018 meta-analysis showed that of all LGBTQ+ health topics, transgender care was reported to be the most poorly understood by medical students. ${ }^{2}$

To expand upon the authors' call for reforming medical school curricula, we caution that the majority of interventions designed to address shortcomings in transgender health education were one-time attitude- and awareness-based interventions that showed short-term improvement but lacked long-term efficacy. ${ }^{2}$ We therefore stress the importance of integrating TNB health topics within existing preclinical and clinical teaching frameworks - rather than having them siloed off in elective or optional courses.

As examples of successful curricular integration, the University of Louisville School of Medicine developed eQuality in 2016, incorporating $50 \mathrm{~h}$ of LGBTQ + health directly into the preclinical curriculum. ${ }^{3}$ Baylor College of Medicine teaches LGBTQ + health topics under the umbrella of social determinants of health and intersectionality, whereas the Vagelos College of Physicians \& Surgeons at Columbia University addresses it through the lens of cultural humility. ${ }^{3}$ All of these initiatives not only increased students' knowledge of LGBTQ + health topics, but also improved their confidence in caring for an LGBTQ + patient population. ${ }^{3}$

A model for integrated clinical training can be found at the Medical University of South Carolina, where clinical students participated in a weekly seminar during their psychiatry clerkship. ${ }^{4}$ The objectives of these seminars were to recognize the importance of LGBTQ-specific education in a clinical setting, to build a foundation for LGBTQ + terminology and using inclusive language, and to discuss how

Prior presentations: None.

Received: 7 August 2021

Accepted: 29 October 2021

Published online January 7, 2022 insensitive communication can lead to adverse health outcomes. The seminars included didactic lectures, multiple choice questions, and clinical vignettes, and discussed topics such as mental health disorders and gender minority stress within the LGBTQ + community. We propose that a similar TNB-focused initiative based in the surgery clerkship might discuss indications and counseling for gender-affirming surgeries, while another in the OB/GYN clerkship might outline fertility preservation or cancer risks for TNB individuals.

We thank the authors for their expertise in this field and welcome innovative approaches to dismantle the structural and interpersonal barriers to TNB and LGBTQ+healthcare rooted in our medical school curricula.

Christopher K. Wong, BA, BS ${ }^{1}$

Shefali P. Jain, BS ${ }^{2}$

Matthew Van de Graaf, $\mathrm{BS}^{3}$

${ }^{1}$ School of Medicine, Baylor College of Medicine, Houston, TX, USA

${ }^{2}$ Medical College of Georgia, Augusta, GA, USA

${ }^{3}$ Eastern Virginia Medical School, Norfolk, VA, USA

Corresponding Author: Christopher K. Wong, BA, BS; School of Medicine, Baylor College of Medicine, Houston, TX, USA (email: ckwong@bcm.edu)

\section{Declarations}

Conflict of Interest The authors have no relevant financial or non-financial interests to disclose.

\section{References}

1. Warner DM, Mehta AH. Identifying and addressing barriers to transgender healthcare: where we are and what we need to do about it. J Gen Intern Med. 2021.

2. Dubin SN, Nolan IT, Streed CG, Greene RE, Radix AE, Morrison SD. Transgender health care: improving medical students' and residents' training and awareness. Adv Med Educ Pract. 2018;9:377-391.

3. O'Leary KB, Kunkel GH. Restructuring LGBTQ curriculum in medical schools. Acad Psychiatry. 202 1;45:487-490.

4. Fadus MC, Peterson NK, Jilich CL, et al. Improving communication with LGBTQ patients: a pilot curriculum during the psychiatry clerkship. Acad Psychiatry. 2020;44:218-222.

Publisher's note Springer Nature remains neutral with regard to jurisdictional claims in published maps and institutional affiliations. 